\title{
Performance Study of Global Weight Window Generator Based on Particle Density Uniformity
}

\author{
Peng He ${ }^{1}$, Bin WU ${ }^{1}$, Lijuan HAO ${ }^{1}$, Guangyao SUN ${ }^{1}$, Bin LI ${ }^{1}$, and Ulrich FISCHER ${ }^{2}$ \\ ${ }^{1}$ Key Laboratory of Neutronics and Radiation Safety, Institute of Nuclear Energy Safety \\ Technology, Chinese Academy of Sciences \\ Hefei, Anhui, 230031, China \\ ${ }^{2}$ Karlsruhe Institute of Technology (KIT) \\ Hermann-von-Helmholtz-Platz 1, 76344, Eggenstein-Leopoldshafen, Germany \\ peng.he@fds.org.cn, bin.wu@fds.org.cn, lijuan.hao@fds.org.cn, guangyao.sun@fds.org.cn, \\ bin.li@fds.org.cn, ulrich.fischer@kit.edu
}

\begin{abstract}
The variance reduction techniques are necessary for Monte Carlo calculations in which obtaining a detailed calculation result for a large and complex model is required. The GVR method named as global weight window generator (GWWG) was proposed by the FDS team. In this paper, two typical calculation examples, ISPRA-Fe benchmark in SINBAD (Shielding Integral Benchmark Archive Database) and TF Coils (Toroidal Field coils) of European HCPB DEMO (Helium Cooled Pebble Bed demonstration fusion plant), are used to study the performance of GWWG method. It can be seen from the calculation results that the GWWG method has a significant effect in accelerating the Monte Carlo calculation. Especially when the global convergence calculation results are needed, the acceleration effect $\left(\mathrm{FOM}_{\mathrm{G}}\right)$ can reach $10^{5}$ or more. It proves that the GWWG method is an effective tool for deep-penetration simulations using Monte Carlo method.
\end{abstract}

KEYWORDS: SuperMC, Global variance reduction, Monte Carlo, Particle transport

\section{INTRODUCTION}

Monte Carlo (MC) is a high precision simulation method which is widely used in particle transport simulation. In MC method, the transport process of particle in phase space is simulated using the principle of random sampling. Therefore, in the deep-penetration shielding calculation, a large number of particles must be simulated to ensure that there are enough particles reached the tally area. It costs huge amount of computing resources. It is a challenge to improve computational efficiency to get reliable results in as little time as possible for deep-penetration shielding cases in the MC simulation.

MC transport codes usually use variance reduction (VR) methods to obtain sufficient precise results in acceptable run time. VR methods bias more particles to important phase spaces, which helps to improve the convergence speed of the tally results. Meanwhile, the particle weight is adjusted accordingly to ensure that the tally results are unbiased. Up to now, several VR methods, such as geometry and energy splitting or roulette, dxtran sphere, exponential transform and weight window and so on, have been developed. One of the most efficient VR method is the weight window method. The weight window 
method uses the weight window parameter to control particle splitting or roulette, thereby biasing the particles to the interesting areas. The high quality weight window parameters could effectively reduce the statistical error in target region. By setting appropriate weight window parameters, it is possible to uniformly sample particles in the whole phase space, thus achieving a global variance reduction (GVR).

There are two well-known GVR methods now, Consistent Adjoint Driven Importance Sampling (CADIS) method proposed by John C. Wagner et al. [1] - [3], and MAGIC method proposed by Andrew Davis et al. [4] and A.J. van Wijk et al. [5]. In CADIS method, the source biasing factors and the global weight window parameters are determined based on the adjoint flux obtained from additional $\mathrm{S}_{\mathrm{N}}$ calculations. In MAGIC method, the global weight window parameters are generated based on the mesh forward flux obtained by several iterations of MC calculations. For CADIS, due to an additional $\mathrm{S}_{\mathrm{N}}$ calculation, it may result in a large effort in creating the $S_{N}$ neutronics model for a large and complex model such as ITER. And for MAGIC method, although it uses the same model in generation of weight window and formal calculation, the long-history of some particles will increase the computation time.

An innovative GVR method - global weight window generator (GWWG) based on the particle density uniformity, has been proposed by the FDS team [6]. The GWWG method has been implemented in the Super Multi-functional Calculation Program for Nuclear Design and Safety Evaluation (SuperMC) [7]-[8] which is a large-scale integrated software system for neutronics design. SuperMC can be used for the design and safety evaluation of nuclear systems, as well as nuclear technology application field including radiation medicine, nuclear detection and so on.

This paper will use the GWWG method to perform two deep-penetration shielding application calculations and analyze the acceleration performance of the GWWG method.

\section{Methodology}

The GWWG $[9,10]$ method adopts several basic ideas of WWG such as the importance of phase space cells and the contribution feedback along the particle's trajectory. Instead of a local variance reduction, the GWWG can achieve a global variance reduction. In particular, the concept of particle density uniformity is introduced, and the importance is defined as the contribution to global particle density uniformity [11]:

$$
\text { Importance }=\frac{C_{i}}{W_{i}}
$$

Where $C_{i}$ is the total contribution to particle density uniformity, which is accumulated from the simulation steps of every particle, $W_{i}$ is the total weight of particles entering the cell.

Two step Monte Carlo calculations are required when using the GWWG method. The first one is performed to get the importance of meshes in whole space, and then accurate values of weighted window parameters and generate the weight window file. The second one is performed using the weight window file to obtain the tally results. The weight window generation process is divided into fixed length iteration steps, and the weight window is updated at the end of each iteration step.

\section{RESULTS AND DISCUSSIONS}

In this work, two typical deep-penetration cases in both fission and fusion applications, the ISPRA-Fe benchmark case and the radiation loads on TF Coils in European HCPB DEMO, have been calculated with SuperMC using GWWG method to show the performance of GWWG method. 


\subsection{ISPRA-Fe benchmark}

The ISPRA-Fe benchmark example belongs to the ISPRA series benchmark examples. The ISPRA series benchmark examples from the shielding integral benchmark archive and database (SINBAD) released by OECD/NEA and Radiation Safety Information Computational Center (RSICC), are typical deep-penetration shielding experiments. They are used to verify the validity and correctness of nuclear cross-section libraries and computational codes in deep-penetration shielding calculation. 1

The experiment was carried out on the EURACOS II (Enriched URAnium Convertor Source) as shown in Fig. 1. The EURACOS II consists of three parts: neutron source, shield and detector. The shield component is an iron mock-up, which is a block of dimensions $145 \times 145 \times 130 \mathrm{~cm}^{3}$. Several detectors are placed at different thicknesses of the shield along the axis of iron block. The geometry model built in SuperMC is shown in Fig. 2.

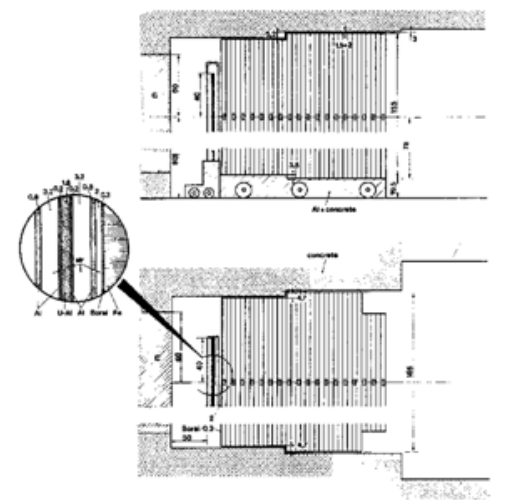

Figure 1. The true model of ISPRA-Fe Experiment.

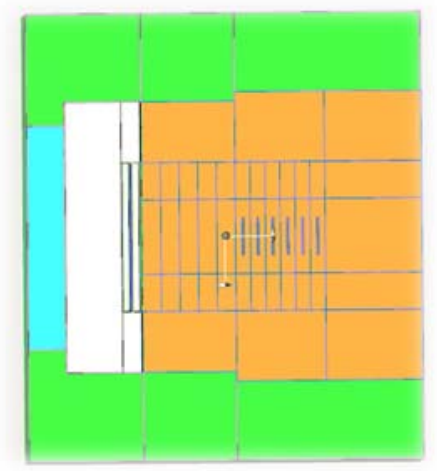

Figure 2. The geometry model built in SuperMC.

Two calculations are performed separately by SuperMC (use GWWG method) and MCNP (analog run, no acceleration technique except implicit capture). Table I shows the calculation results of the reaction rate calculation at the distance of $94 \mathrm{~cm}$ from the source. The figure of merit (FOM) is defined as below:

$$
\mathrm{FOM}=\frac{1}{\sigma^{2} t}
$$

where $\sigma$ is the statistical error, $t$ is total running time.

It can be seen from the results that SuperMC result with GWWG method achieve a good acceleration compared to MCNP analog calculation. 
Table I. The comparison of SuperMC and MCNP calculation results in single location

\begin{tabular}{|c|c|c|c|c|}
\hline & Run Time (min) & Error (\%) & FOM & Speedup $^{(*)}$ \\
\hline MCNP Analog & 3350.7 & 13.82 & $1.563 \mathrm{E}-02$ & 1 \\
\hline SuperMC & 1543.9 & 0.634 & 16.41 & 1049 \\
\hline
\end{tabular}

*The Speedup $=$ the FOM of the SuperMC Calculation / the FOM of the MCNP Analog Calculation

Then the weight window parameter is used to perform $\mathrm{MC}$ calculations to get the global reaction rates in every mesh. In this calculation, the tally area is divided into $40 * 50 * 50$ meshes. The calculation results are shown in Table II, with the results of analog run for comparison. For the GWWG case, the average statistical error is $0.583 \%$, while for the analog run case, the average statistical error is $44.6 \%$. The global figure of merit $\left(\mathrm{FOM}_{\mathrm{G}}\right)$ is defined as below:

$$
\mathrm{FOM}_{G}=\frac{1}{t \sum_{n=1}^{N} \sigma^{2} / N}
$$

where $t$ is total running time, $\sigma$ is the statistical error of the $n$-th mesh, and $N$ is the total number of meshes.

In the calculation of the global reaction rate calculation, the speedup factor in global $\mathrm{FOM}_{\mathrm{G}}$ is 12709 compared with the analog run. Fig. 3 shows the maps of the reaction rate and statistical error. It could be seen that the GWWG method could significantly improve the convergence speed of the global calculation results.

Table II. Comparison of GWWG and Analog calculation results of the global reaction rate calculation

\begin{tabular}{|l|c|c|c|c|}
\hline & Run Time $(\mathrm{min})$ & Error $(\%)$ & $\mathrm{FOM}_{\mathrm{G}}$ & Speedup \\
\hline SuperMC-Analog & 45545 & 44.6 & $1.103 \mathrm{e}-4$ & 1 \\
\hline SuperMC-GWWG & 21024 & $5.83 \mathrm{e}-1$ & 1.402 & 12709 \\
\hline
\end{tabular}

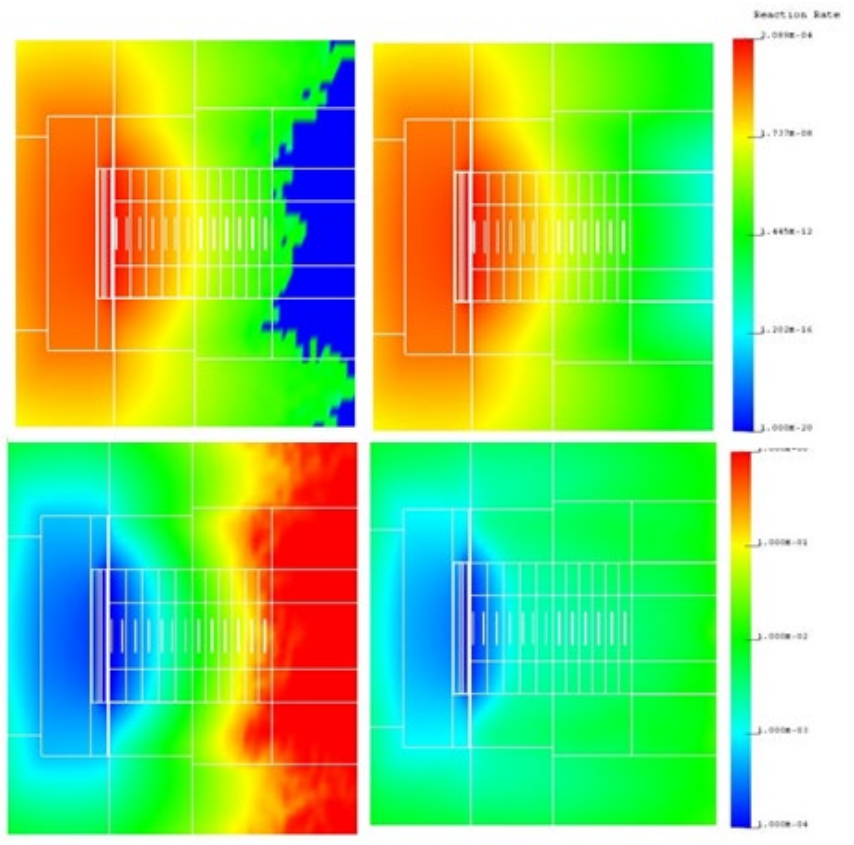


Figure 3. Reaction rate (top) and statistical error (bottom) maps obtained using analog (left) and GWWG method (right).

\subsection{TF Coils of European HCPB DEMO}

In the European DEMO, the shielding between inboard blankets and TF coils is the weakest, so the main attention on radiation loads on coils is focused on inboard TF coils. The nuclear heating density, fast neutron flux and displacement damage to copper on the slice of TFC closest to inboard blankets were estimated by the two codes. To calculate radiation loads distributions on TFC, the TFC cell (marked by yellow pane in Fig.4 left) was sliced into smaller 72 cells with thickness of $\sim 5.0 \mathrm{~cm}$ and tallied accordingly as shown in Fig.4 right.

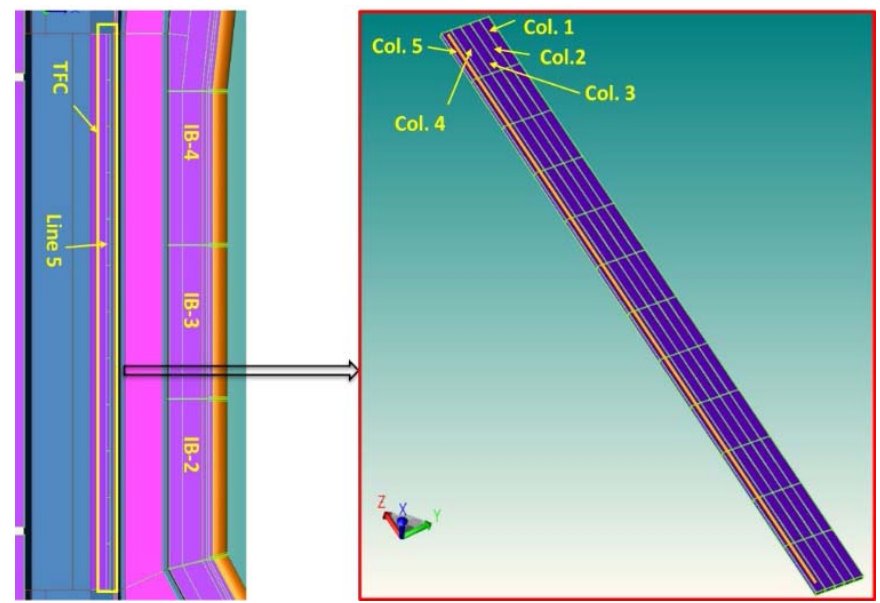

Figure 4. Tally cells for radiation loads on TFC

In calculating radiation loads on TFC, a global weight window generated by SuperMC using GWWG method was applied to obtain results with reasonably low statistical error around TFC region (thick shielding region). The efficiency of global weight window was evaluated by comparing Figure of Merits (FOMs) of biased (with weight window via GWWG) and analog (without weight window) Monte Carlo calculations carried out on the same clusters.

And for case in which both tally result and statistical error are zero, the corresponding statistical error is regarded as $100 \%$ for calculating FOM. Fig.5 shows the distribution of fast neutron flux with statistical error on TF coil in cases of analog (without weight window) and biased (with weight window via GWWG) calculations performed by SuperMC. As shown in Tab. III, it was found that the simulation with global weight window generated via GWWG was speeded up by $~ 164$ times compared to analog simulation in term of FOM. 


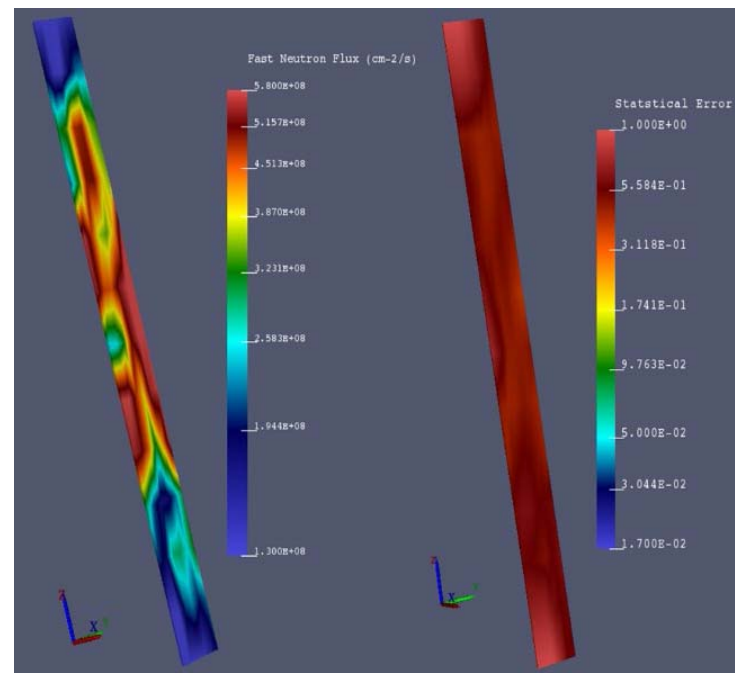

(a) Analog

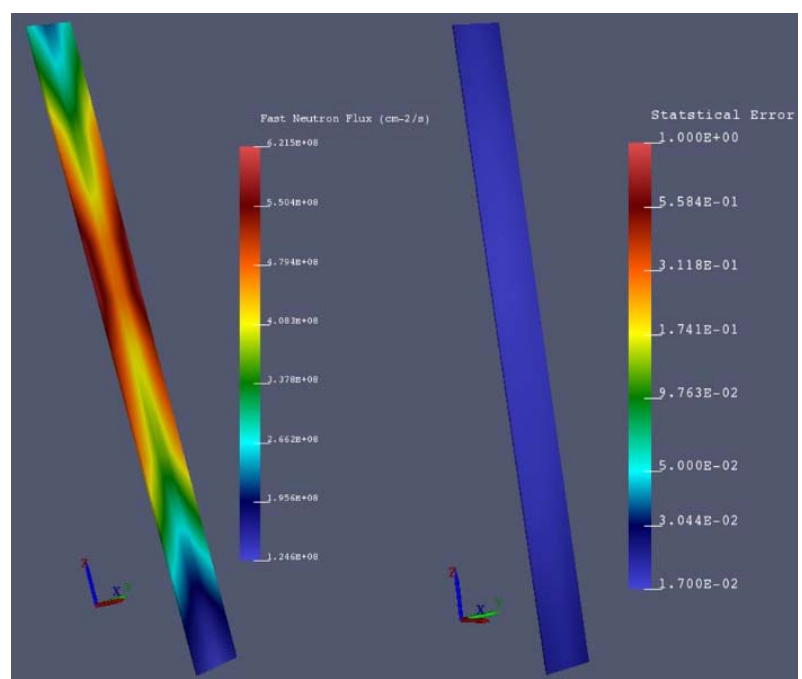

(b) GWWG

Figure 5. Map of fast neutron flux with statistical error on TF coil calculated by SuperMC

Table III. FOMs of Analog and GWWG calculations performed by SuperMC

\begin{tabular}{|l|c|c|c|c|}
\hline & Run Time (min) & Error (\%) & FOM $_{G}$ & Speedup \\
\hline Analog & 111427 & $3.56 \mathrm{E}-01$ & $2.52 \mathrm{E}-05$ & 1 \\
\hline GWWG & 653548.8 & $3.70 \mathrm{E}-04$ & $4.13 \mathrm{E}-03$ & 163.9 \\
\hline
\end{tabular}

\section{CONCLUSIONS}

In this paper, two typical deep-penetration cases, ISPRA-Fe benchmark in SINBAD and TF Coils of European HCPB DEMO, are used to study the performance of GWWG method. In ISPRA-Fe benchmark, the GWWG method achieves 1049 times acceleration in single position reaction rate calculation compared with the MCNP analog run, and 12709 times acceleration in global reaction rate calculation, compared with SuperMC simulation without GWWG method. In calculation of radiation loads on TF Coils of European HCPB DEMO, the GWWG method achieves 163.9 times acceleration comparing to SuperMC simulation without GWWG method. It proves that the GWWG method is an effective tool for deep-penetration cases.

\section{ACKNOWLEDGMENTS}

Part of the MCNP and SuperMC calculations leading to the results of this work were performed on the ForHLR II computer cluster at KIT funded by the Ministry of Science, Research and the Arts Baden-Württemberg and DFG ("Deutsche Forschungsgemeinschaft"). The authors would like to show their great appreciation to other members of the FDS Team for supports to this research.

\section{REFERENCES}

1. Y. Wu, Z. Chen, L. Hu, et al., Identification of safety gaps for fusion demonstration reactors [J], Nature Energy, 2016 (1): 16154.

2. Wagner J C, Acceleration of Monte Carlo shielding calculations with an automated variance reduction technique and parallel processing [D]. Pennsylvania State University, 1997. 
3. J.C. Wagner, E.D. Blakeman, D.E. Peplow, Forward-weighted CADIS method for global variance reduction [J], Transaction of the American Nuclear Society, 2007 (97): 630-633.

4. Van Wijk A J, Van den Eynde G, Hoogenboom J E. An easy to implement global variance $r$ eduction procedure for MCNP [J]. Annals of Nuclear Energy, 2011, 38(11): 2496-2503.

5. A. Davis, A. Turner, Comparison of global variance reduction techniques for Monte Carlo radiation transport simulations of ITER [J], Fusion Engineering and Design, 2011, 86(9-11), 2698-2700.

6. A.J. van Wijk, G. Van den Eynde et al., An easy to implement global variance reduction procedure for MCNP [J], Annals of Nuclear Energy, 2011(38): 2496-2503.

7. $\mathrm{Y} \mathrm{Wu}$, Multi-functional neutronics calculation methodology and program for nuclear design and radiation safety evaluation [J], Fusion Science and Technology, 2018, 74(4): 321-329.

8. Y Wu, CAD-Based Interface Programs for Fusion Neutron Transport Simulation [J], Fusion Engineering \& Design, 2009, 84 (7-11):1987-1992.

9. Y Wu, J Song, $\mathrm{H}$ Zheng, et al., CAD-Based Monte Carlo Program for Integrated Simulation of Nuclear System SuperMC [J], Annals of Nuclear Energy, 2016 (82):161-168.

10. Zhang S, Yu S, He P, Verification of SuperMC with ITER C-Lite Neutronic Model [J], Fusion Engineering and Design, 2016 (113): 126-130.

11. Y Wu, S Zhang, J Song, et al. A Global Weight Window Parameter Generation Method Based on Particle Density uniformity for Monte Carlo Calculation [P]. China Patent: CN106295213A, 2017. ( http://cpquery.sipo.gov.cn/) 